\title{
RELATION BETWEEN LIGHT VARIATIONS OF SOLAR SYSTEM SATELLITES AND THEIR INTERACTION WITH INTERPLANETARY MEDIUM
}

\author{
C. BLANCO and S. CATALANO \\ Osservatorio Astrofisico di Catania, Istituto di Astronomia dell'Università di Catania, Italy
}

\begin{abstract}
The behaviour of the available light curves of Jupiter and Saturn satellites is shown to change steadily from inner to outer satellites.

The change in reflectivity of satellites with orbital motion could be explained assuming that dark meteoroidal material accumulates on a given portion of the satellite's surface, which was once mainly covered with ice or snow. The preferential impact location on the satellite's surface could be a consequence of the perturbing action of the planet on the motion of interplanetary material.
\end{abstract}

\section{Introduction}

Periodic intrinsic light variations are known for many satellites in the solar system (Harris, 1961). These variations give information about the diffuse reflection properties of satellite surfaces or atmospheres, about their interaction with the interplanetary medium, and the rotation about their own axes. The light variation periods of satellites are equal to their orbital periods. This fact suggests that the rotation and revolution periods are the same. In the Jupiter and Saturn systems, the maximum of the light curve shifts from near eastern elongation, for the inner satellites, to western elongation for the outer ones.

Observations of the Saturn satellites Rhea and Titan and of the Galilean satellites of Jupiter were carried out at the Catania Observatory (Blanco and Catalano, 1971, 1974) to investigate a possible mechanism responsible for the different reflecting properties of the leading and trailing hemispheres for the inner and outer satellites.

Spectral reflectivity measurements have led to the suggestion that Saturnian satellite surfaces are composed primarily of ice (Kuiper, 1952). The four large, bright Jupiter satellites are also partially covered with ice or water frost (Kuiper, 1952; Sky Telesc., 1973). However, the reddish colour observed for many satellites indicates that there would have to be impurities in the frost or ice.

In order to explain these results we make the hypothesis that the light variations of satellites are due to erosion or accumulation of dark meteoroidal material on a portion of their surfaces once covered with ice, taking into account the perturbing action of the planet on the orbits of interplanetary material.

\section{Discussion}

The best available light-curves in the $V$ band of the main Jupiter and Saturn satellites are displayed in Figure 1. In this figure, data are corrected for the solar phase angle effect, and orbital phase angles $\theta$ are given according to synodical periods, starting 


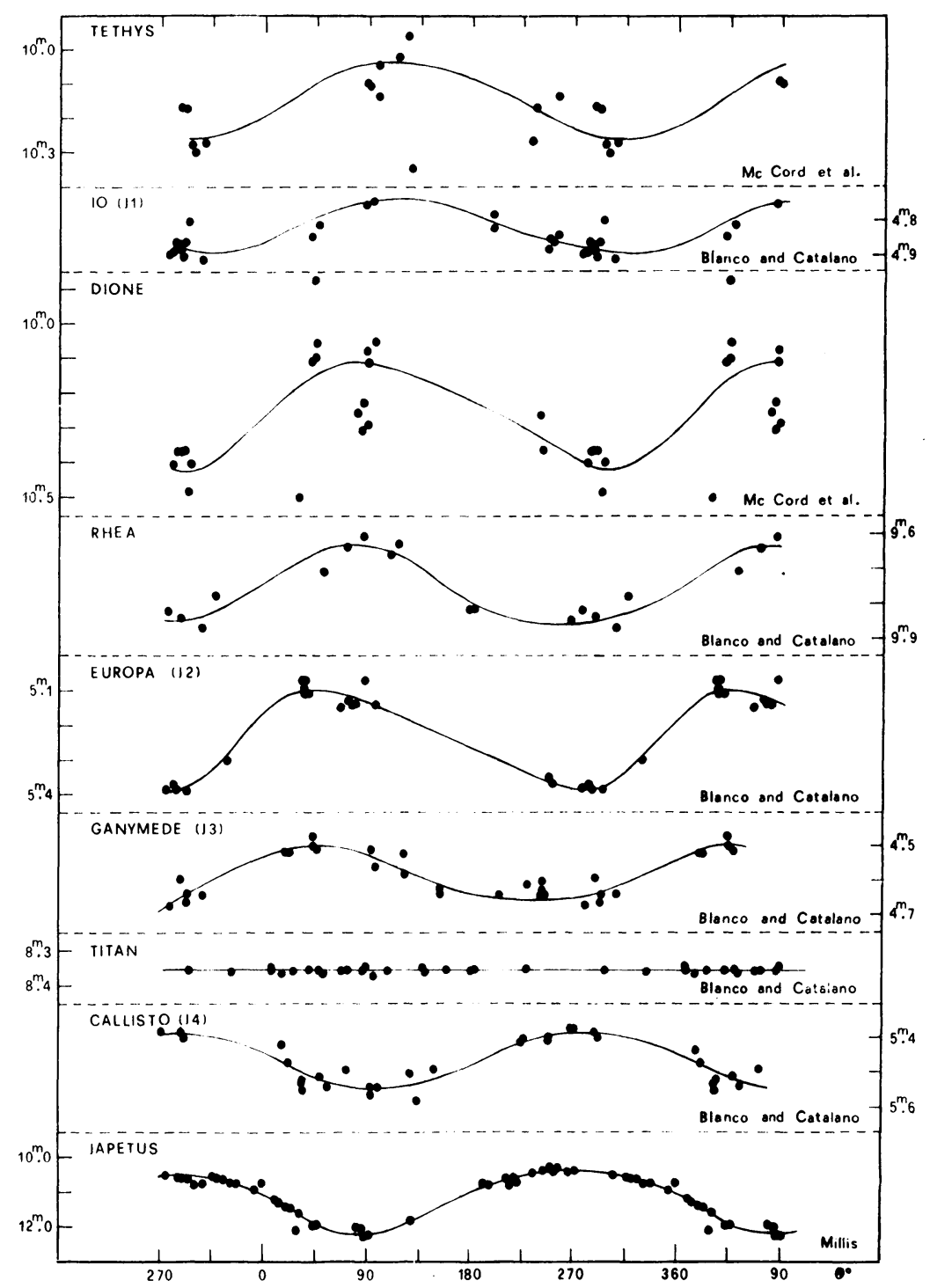

Fig. 1. Light curves in the $V$ band for Jupiter and Saturn satellites.

from the geocentric superior conjunction. Since the satellites are synchronous, as indicated by their light curves, there will be a one-to-one correspondence between $\theta$ and the longitude of the sub-Earth point on the satellite's surface. The rotational phase angle will hereafter be indicated by the corresponding orbital phase angle. In Figure 1 the light-curves of satellites are given from top to bottom in order of increasing distance from the planet; a shift of the orbital phase angle of minimum (or maximum) light is clearly visible. Among all the well-observed satellites in Figure 1, only Titan shows no variation of brightness with orbital phase. This is not surprising, 
TABLE I

Distance from the primary expressed in units of the planet's radius, average rotational phase angle of maximum and minimum light, and number of estimations for Jupiter and Saturn satellites

\begin{tabular}{|c|c|c|c|c|c|}
\hline \multirow[t]{2}{*}{ Satellite } & \multirow[t]{2}{*}{$d$} & \multicolumn{2}{|c|}{$\begin{array}{l}\text { Average rotational } \\
\text { phase angle }\end{array}$} & \multirow[t]{2}{*}{$n$} & \multirow[t]{2}{*}{ References } \\
\hline & & $\theta_{\max }$ & $\theta_{\min }$ & & \\
\hline Tethys & 4.9 & $120^{\circ}$ & $310^{\circ}$ & 1 & $\begin{array}{l}\text { McCord et al. (1971) } \\
\text { (Blanco and Catalano (1974) }\end{array}$ \\
\hline $\operatorname{Io}(J 1)$ & 5.9 & 143 & 328 & 3 & $\left\{\begin{array}{l}\text { Harris (1961) } \\
\text { Johnson (1971) }\end{array}\right.$ \\
\hline Dione & 6.25 & 60 & 290 & 1 & $\begin{array}{l}\text { McCord et al. (1971) } \\
\text { Blanco and Catalano (1971) }\end{array}$ \\
\hline Rhea & 8.8 & 60 & 250 & 3 & 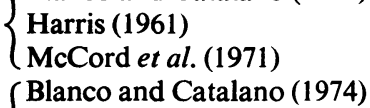 \\
\hline Europa $(J 2)$ & 9.4 & 78 & 287 & 3 & $\begin{array}{l}\text { Harris (1961) } \\
\text { Johnson (1971) }\end{array}$ \\
\hline Ganimede $(J 3)$ & 15 & 50 & 223 & 3 & $\begin{array}{l}\text { Harris (1961) } \\
\text { Johnson (1971) }\end{array}$ \\
\hline Callisto (J4) & 26.4 & 280 & 143 & 3 & $\begin{array}{l}\text { Harris }(1961) \\
\text { Johnson }(1971)\end{array}$ \\
\hline Japetus & 59.7 & 270 & 90 & 3 & $\left\{\begin{array}{l}\text { McCord et al. }(1971) \\
\text { Sky Telesc. }(1973)\end{array}\right.$ \\
\hline
\end{tabular}

since Titan has an atmosphere. The rotational phase angles of minimum and maximum light, averaged from estimations made on the available light curves, are given in Table I for each satellite, together with mean distance from the planet, expressed in units of the planet's radius. The averaged values of $\theta_{\min }$ and $\theta_{\max }$ vs the distance are plotted in Figure 2. From this figure it appears that the phase shift of the maximum and minimum light with distance from the planet is quite regular. The data are fitted quite well by the following simple relation

$$
\theta=K \cdot d^{-1 / 2}+K^{\prime}
$$

represented in Figure 2 by the line obtained for $K=833.3$ and $K^{\prime}\left(\theta_{\min }\right)=-16: 7$, or $K^{\prime}\left(\theta_{\max }\right)=163$ :3.

The values of $\theta$ corresponding to the minimum light fit the above relation better than the maximum ones. We note that the relation (1) seems to be independent of the particular planet-satellite system. In fact the data for the Jupiter and Saturn satellites, all represented in the Figure 2, agree quite well with one another.

Light variations of Japetus, which appears darker in the leading hemisphere, have been explained by an erosion process resulting from interplanetary impacts of meteoroids on the leading hemisphere once covered with ice or water frost (Cook and Franklin, 


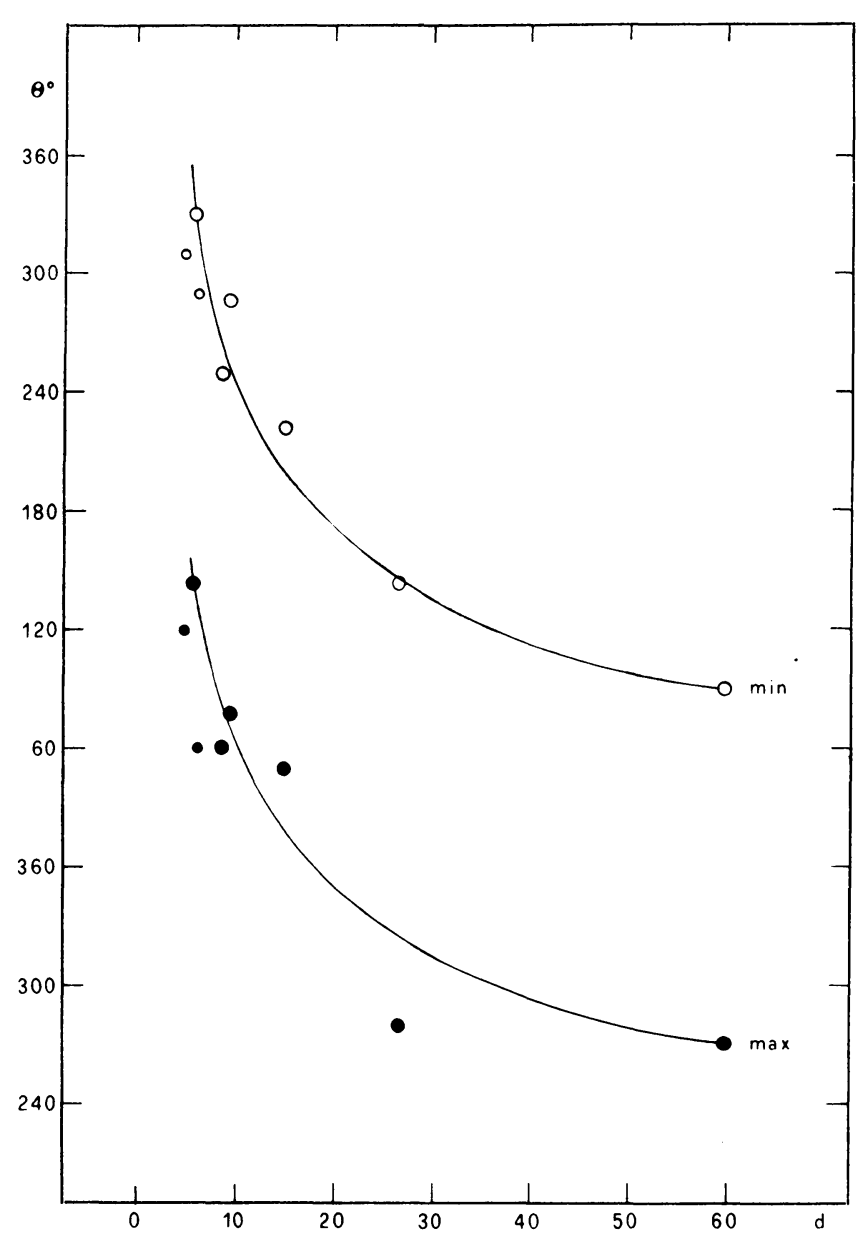

Fig. 2. Average rotational phase angle of maximum and minimum light for Jupiter and Saturn satellites vs. distance from the primary, expressed in units of the planet's radius. The size of dots indicates the number of estimations on different authors' light curves. The lines were computed according to formula (1).

1970). Such an interpretation cannot explain the behaviour of light variations for inner satellites which, as they appear darker in the trailing hemisphere, would suffer more impacts in this hemisphere, in spite of their higher orbital velocity.

Taking into account planetary perturbations on the interplanetary material, the regular shift of $\theta_{\min }\left(\theta_{\max }\right)$ with distances in Figure 2 might be better explained by meteoroidal impact mechanism, in which the distance from the planet plays the main role. Meteoroidal material entering the planet's sphere of influence will describe open hyperbolic orbits, may be captured in elliptic unstable orbits, or may become, if they loose energy by impact or drag, permanent stable satellites of the planet, thus increasing the dust density near the planet. Figure 3 shows a schematic representation of trapped and non-trapped particles near the planet, in direct and retrograde orbits. The regular 


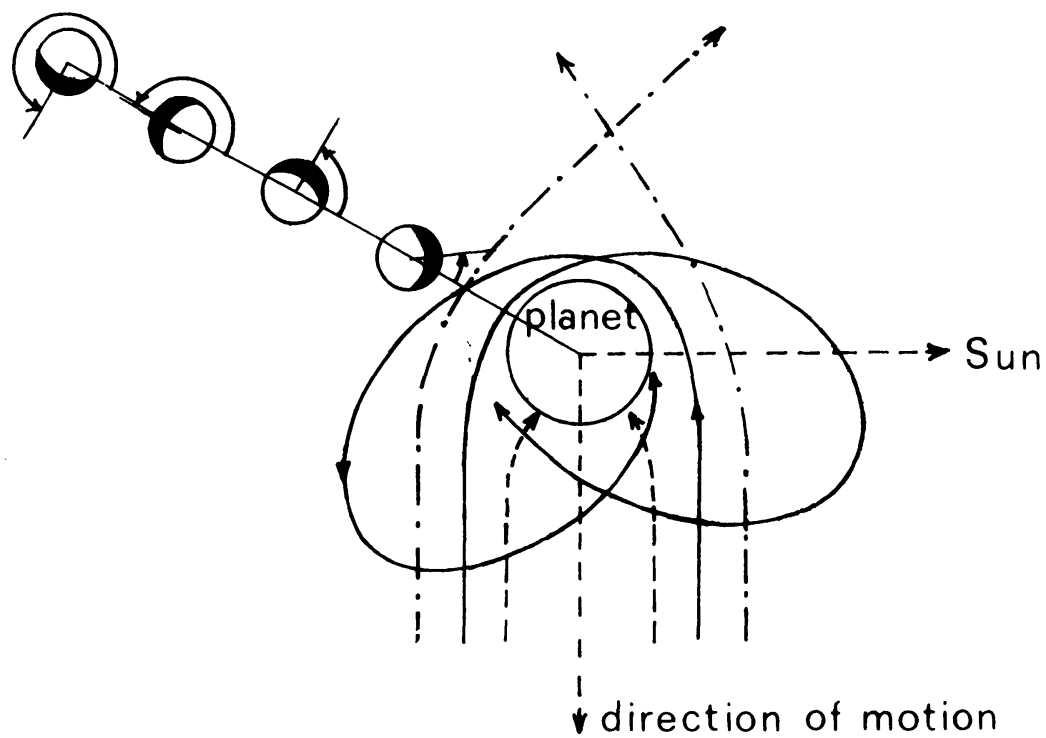

Fig. 3. Schematic representation of orbits of trapped and non-trapped particles in the sphere of influence of a planet. The darked part of satellites' surfaces represents the location of low reflectivity. $\theta$ is the rotational phase angle of minimum light according to the light curves.

shift of $\theta_{\min }\left(\theta_{\max }\right)$ with the distance may be better understood in terms of impacts on the satellite surface by particles mainly moving in direct, high-eccentricity orbits. Some interplanetary particles, when within the planet's sphere of influence, will impinge on the satellite surfaces. The correlation between rotational phase angle of minimum light of the satellite and distance from the planet might be explained, bearing in mind that the location of impacting material on the satellite surface depends on the particlesatellite relative vectorial velocity, which in turn depends on the $V_{\infty}$ velocity vector and on the distance of the incoming asymptote. We note that the orbital velocities of both satellites and dust particles depend on the inverse square root of distance from the planet, so the relative velocity would show the same dependence on $d$. If so, a correlation follows between the impact velocity of the dust particles and the rotational phase of minimum reflectivity, that is the satellite rotational angle of the greatest meteoroid accumulation.

\section{Conclusion}

The light variation of Jupiter and Saturn satellites could be interpreted in terms of meteoroidal material accumulation on a given part of their surface.

The constancy of Titan could be explained by taking into account its atmosphere. The light is mainly diffused by the atmosphere, which has a more uniform reflectance than we can expect from solid surfaces. In addition, the atmosphere could also brake the interplanetary impacts so that the surface features of the leading and trailing hemispheres are not greatly different. 
However, a more detailed analysis is needed. In a model of capture of interplanetary medium by the planets, the orbital dynamics of particles affected has to be carefully considered to explain the correlation between the impact velocity of the dust particles and the rotational angle of minimum reflectivity.

In this context, detailed analysis of data on particle orbits obtained by means of space probes in the Earth-Moon system could be very useful.

\section{References}

Blanco, C. and Catalano, S.: 1971, Astron. Astrophys. 14, 43.

Blanco, C. and Catalano, S.: 1974, Astron. Astrophys. (in press).

Cook, A. F. and Franklin, F. A.: 1970, Icarus 13, 282.

Harris, D. L.: 1961, in G. P. Kuiper and B. M. Middlehurst (eds.), Planets and Satellites, The University of Chicago Press, Chicago, p. 272.

Johnson, T. V.: 1971, Icarus 14, 94.

Kuiper, G. P. (ed.): 1952, The Atmospheres of Earth and Planets, The University of Chicago Press, Chicago, p. 306.

McCord, T. B., Johnson, T. V., and Elias, J. H.: 1971, Astrophys. J. 165, 413.

Sky Telesc.: 1973, 45, 22.

\section{DISCUSSION}

Dennefeld: If the Galilean satellites are covered with ice, the thermal velocity of evaporated water molecules is not sufficient for allowing escape from the satellite. Then, the water molecules follow ballistic orbits and then stick onto surface. This gives evidence for the existence of a layer of water vapor, with thickness of the order of $70 \mathrm{~km}$ and density of the order of $10^{7} \mathrm{H}_{2} \mathrm{O} \mathrm{cm}$, if the temperature at the surface is $130 \mathrm{~K}$. The density can be increased by $10^{4}$ if hydrated ammonia is present as suggested by $J$. Lewis. Furthermore, these molecules can be destroyed by charged particles, and this can explain the absence of ice on Io.

Baum: Astronomers who have an opportunity to obtain light curves of the Jovian satellite events and who would like their results to be included in a combined analysis are invited to send a copy of their light curves to Dr Robert Milis at Lowell Observatory. Timing accuracy, to one second or better, is needed. 